Accepted for publication in The Astrophysical Journal

Preprint typeset using $\mathrm{LATEX}_{\mathrm{E}}$ style emulateapj v. 11/10/09

\title{
STRUCTURE IN THE ROTATION MEASURE SKY
}

\author{
J. M. Stil, A. R. TAYlor, and C. Sunstrum \\ Institute for Space Imaging Science and Department of Physics and Astronomy, University of Calgary \\ Draft version March 22, 2022
}

\begin{abstract}
An analysis of structure in rotation measure (RM) across the sky based on the RM catalog of Tavlor et al. (2009) is presented. Several resolved RM structures are identified with structure in the local ISM, including radio loops I, II, and III, the Gum nebula, and the Orion-Eridanus super bubble. Structure functions (SFs) of RM are presented for selected areas, and maps of SF amplitude and slope across the sky are compared with $\mathrm{H} \alpha$ intensity and diffuse polarized intensity. RM variance on an angular scale of $1^{\circ}$ is correlated with length of the line of sight through the Galaxy, with a contribution from local structures. The slope of the SFs is less concentrated to the Galactic plane and less correlated with length of the line of sight through the Galaxy, suggesting a more local origin for $\mathrm{RM}$ structure on angular scales $\sim 10^{\circ}$. The RM variance is a factor $\sim 2$ higher towards the SGP than towards the NGP, reflecting a more wide-spread asymmetry between the northern and southern Galactic hemispheres. Depolarization of diffuse Galactic synchrotron emission at latitudes $<30^{\circ}$ can be explained largely by Faraday dispersion related to small-scale variance in RM, but the errors allow a significant contribution from differential Faraday rotation along the line of sight.
\end{abstract}

Subject headings: ISM: magnetic fields, ISM: structure, radio continuum: ISM

\section{INTRODUCTION}

The magnetic field of the Milky Way Galaxy is the only galactic magnetic field that can be studied over $\sim$ 5 orders of magnitude, on length scales less than a parcsec to several kiloparsec. The structure of this magnetic field is revealed by different observing techniques that are usually sensitive to either the line-of-sight component of the magnetic field (Faraday rotation, Zeeman splitting), or the component of the magnetic field perpendicular to the line of sight (stellar polarization, polarization of radio synchrotron emission). Zeeman splitting provides a direct in-situ measurement of the strength of the magnetic field, while Faraday rotation and stellar polarization are propagation effects that require additional information on the electron density or the extinction column of dust along the line of sight. Galactic synchrotron emission at frequencies of a few $\mathrm{GHz}$ suffers from strong depolarization from Faraday rotation in the Galactic disk (e.g. Sokoloff et al. 1998; Gaensler et al. 2001; Uyaniker et al. 2003). Even at high frequencies (e.g. $20 \mathrm{GHz}$ WMAP; Page et al. 2007) where Faraday rotation is not significant, the summation of polarized emission with different polarization angles over a long line of sight can be a significant source of depolarization.

The large-scale structure of the Galactic magnetic field has been revealed mostly by Faraday rotation of polarized emission from pulsars and distant active galactic nuclei (AGN) (Simard-Normandin \& Kronberg 1979, 1980; Han et al. 2006; Brown et al. 2001, 2003, 2007; Vallée 2008; Men et al. 2008; Taylor et al.|2009, among others). The angle change $\Delta \theta$ from Faraday rotation of polarized emission of a compact radio source with negligible internal Faraday rotation is given by

$$
\Delta \theta=\lambda^{2} 0.812 \int_{\text {source }}^{\text {observer }} n_{e} \mathbf{B} \cdot d \mathbf{l}=\lambda^{2} R M
$$

where $\lambda$ if the observing wavelength, $n_{e}$ is the electron density, $\mathbf{B}$ is the magnetic field vector, and $d \mathbf{l}$ is a line of sight segment pointing from the source to the observer so that $R M$ has a positive sign if there is a net magnetic field component along the line of sight in the direction of the observer. The angle change $\Delta \theta$ can be determined from observations of the polarization angle of a radio source as a function of wavelength.

The electron density along the line of sight must be determined independently to derive the strength of the magnetic field. For the large-scale magnetic field of the Galaxy, a model of the distribution of the electron density $n_{e}$ along the line of sight has been derived from pulsar dispersion measures (Cordes \& Lazio 2002). However, for smaller length scales, an electron density distribution must be proposed as an assumption that is largely unconstrained by current observations, as significant angle changes can occur in an ionized region that cannot be detected by its thermal emission (Uvaniker et al. 2003). Correlation between fluctuations in electron density and magnetic field strength can bias the RM upward or downward, depending whether there is a positive correlation or an anti-correlation respectively between thermal electron density and field strength (Beck et al. 2003).

Structure in the Galactic magnetic field on smaller scales has been apparent in polarization data of stars within $1 \mathrm{kpc}$ (e.g. Axon \& Ellis 1976; Mathewson \& Ford 1970; Lerov 1999; Heiles 2000; Berdyugin \& Teerikorpi 2002; Berdyugin et al. 2004, and references therein), and polarization of diffuse Galactic synchrotron emission (e.g. Duncan et al 1997, 1999; Uvaniker et al. 1999; Gaensler et al. 2001; Wolleben et al. 2006; Page et al. 2007; Schnitzeler et al. 2007; Wolleben 2007; Gao et al. 2010, Landecker et al., in prep.). Diffuse polarized emission has the advantage that it provides continuous sampling of a foreground Faraday screen, but the signal is usually a complicated integral of synchrotron emissivity and Faraday rotation over the volume probed by the beam (Sokoloff et al. 1998). Equa- 
tion 1 does not apply in this case, but imaging polarized intensity and polarization angle, and RM synthesis (Burn 1966; Brentiens \& De Bruvn 2005) have revealed structures in diffuse polarized emission (e.g. Grav et al. 1998; Gaensler et al. 2001; Uvaniker et al. 2003; Schnitzeler et al. 2007; De Bruvn et al.|2009).

RM structure functions (SFs) (Simonetti, et al. 1984; Simonetti \& Cordes 1986; Minter \& Spangler 1996) have been used to obtain a statistical description of RM variance as a function of angular scale. At high Galactic latitudes the variance in RM was found to be independent of angular scale (Simonetti, et al. 1984; Sun \& Han 2004). At lower latitudes, RM variance is often observed to increase with angular scale (Simonetti \& Cordes 1986; Simonetti 1992; Sun \& Han 2004), sometimes with a break around an angular scale of $1^{\circ}$ above which the variance remains constant or increases at a slower rate (Haverkorn et al.|2003, 2006; Haverkorn 2007; Haverkorn et al. 2008; Rov et al. 2008). The break in the RM SF is usually interpreted as an outer scale in the turbulence that gives rise to the RM variance. The smallest scale that can be probed depends on the density of RM measurements, and angular resolution of the radio data (Simonetti \& Cordes 1986; Leahv 1987). Gaensler et al. (2005) and Haverkorn et al. (2008) used depolarization of point sources as a measure of RM variations on the angular scale of extragalactic radio sources.

A database of 37,543 RMs that covers $80 \%$ of the sky (Taylor et al. 2009) was constructed recently from the NRAO VLA Sky Survey (NVSS; Condon et al. 1998), using the two frequency bands of the NVSS separated by $70 \mathrm{MHz}$ (center to center). The NVSS RMs are reliable to $|R M|<520 \mathrm{rad} \mathrm{m}^{-2}$. Higher RMs are subject to ambiguities because it was not possible to derive a unique RM using the available constraints and noise in the data. This occurs mainly at low Galactic latitude $\left(|b| \lesssim 5^{\circ}\right)$.

\section{LARGE-SCALE RM STRUCTURES AND THE LOCAL ISM}

Tavlor et al. (2009) showed the signature of the largescale magnetic field in the solar neighborhood in these data including a reversal of the sign of RM across the Galactic plane consistent with a quadrupole magnetic field geometry. Assuming a symmetric electron density, they found that the magnetic field strength south of the Galactic plane is approximately two times the magnetic field strength in the north. Towards the Galactic poles, Taylor et al. (2009) found a positive RM in the north and in the south, also stronger in the south. ? found a significant mean RM in the region around the South Galactic Pole (GPP), but not around the North Galactic Pole (NGP).

Here we discuss RM structures on angular scales $\lesssim 90^{\circ}$ and their relation the local interstellar medium. Figures 1, 2, and 3] show the RM database as circles on a background of $\mathrm{H} \alpha$ intensity (Finkbeiner 2003), diffuse polarized emission at $1.4 \mathrm{GHz}$ (Wolleben et al. 2006), and diffuse X-ray emission (Snowden et al. 1998), respectively. Figure 4 shows a different representation of the RM sky towards the anticenter, excluding data with $|R M|<25 \mathrm{rad} \mathrm{m}^{-2}$, and showing all circles at the same size. This representation emphasizes RM structure with amplitudes of a few tens $\mathrm{rad} \mathrm{m}^{-2}$, but also boundaries where the sign of RM changes.

Three large regions previously identified by
Simard-Normandin \& Kronberg (1980) as regions $\mathrm{A}, \mathrm{B}$, and $\mathrm{C}$ are responsible for most of the structure on angular scales $\gtrsim 30^{\circ}$. These structures are also represented in other all-sky RM images with resolution $\sim 20^{\circ}$ (Frick et al. 2001; Johnston-Hollitt et al. 2004; Dineen \& Coles 2005; Xu et al. 2006). The denser sampling of the present data reveals structure in more detail, and we adopt somewhat different boundaries for regions A and C of Simard-Normandin \& Kronberg (1980) as discussed below. The boundaries adopted here are listed in Table 1 and we will refer to region $\mathrm{A}^{\prime}$ and region $\mathrm{C}^{\prime}$ instead. Region B in Simard-Normandin \& Kronberg (1980) refers to the Gum nebula, and will be discussed as such.

Region A is a large area of negative RM for which Simard-Normandin \& Kronberg (1980) listed the boundaries as $60^{\circ}<l<140^{\circ},-40^{\circ}<b<10^{\circ}$. With the superior sampling of the new data, we see substantial substructure in this region. The most negative $\mathrm{RMs}$ occur at intermediate latitudes in the region $80^{\circ}<l<150^{\circ},-40^{\circ}<b<-20^{\circ}$. Distinctly smaller $\mathrm{RM}$ amplitudes are found in the area $100^{\circ}<l<150^{\circ}$, $-20^{\circ}<b<-5^{\circ}$, except for an area with higher RM amplitudes in the longitude range $120^{\circ}<l<130^{\circ}$ (See also Figure 4). The mean RM in the area $100^{\circ}<l<120^{\circ}$, $-40^{\circ}<b<-20^{\circ}$ is $-67.7 \mathrm{rad} \mathrm{m}^{-2}$, with a standard deviation of $38.5 \mathrm{rad} \mathrm{m}^{-2}$. The mean $\mathrm{H} \alpha$ intensity in this area is $1.8 \mathrm{R}$. The mean $\mathrm{RM}$ in the area $100^{\circ}<l<120^{\circ}$, $-20^{\circ}<b<-5^{\circ}$ is $-32.8 \mathrm{rad} \mathrm{m}^{-2}$ with a standard deviation of $31.9 \mathrm{rad} \mathrm{m}^{-2}$. The mean background $\mathrm{H} \alpha$ intensity in this area, avoiding bright $\mathrm{HII}$ regions is $5 \mathrm{R}$. The section of region $\mathrm{A}$ with higher RM amplitude is therefore separated from the Galactic plane by a region with $\mathrm{RM}$ amplitudes a factor $\sim 2$ lower than those at more negative latitudes, even though the $\mathrm{H} \alpha$ intensity is a factor $\sim 2.5$ higher than at more negative latitudes. Simard-Normandin \& Kronberg (1980) suggested that region A extends across the Galactic plane, to $b=+10^{\circ}$, but this appears to be based on only two sources in their Figure 1, and there is no indication of an extension to positive latitudes in their Figure 2. The data of Simard-Normandin \& Kronberg (1980) sampled the intermediate region with low RM amplitudes very sparsely.

Region A is bordered on the high-longitude side by a line where RM nearly vanishes, and then reverses sign to become positive (e.g. Xu et al. 2006). The boundary with near-zero RM runs approximately at an angle of $45^{\circ}$ inclined to the Galactic equator from $(l, b) \approx\left(145^{\circ},-10^{\circ}\right)$ to $(l, b) \approx\left(170^{\circ},-35^{\circ}\right)$ (Figure 41), consistent with the boundary of Radio Loop II (Berkhuiisen et al. 1973). A spur of bright polarized emission is visible in Figure 2 inside the region with negative RM, while strong depolarization is found at the boundary where $\mathrm{RM}$ changes sign. The $\mathrm{H} \alpha$ image shows $\sim 20^{\circ}$ long filaments with a similar orientation with respect to the Galactic equator, approximately coincidental with the line where RM changes sign.

Region B of Simard-Normandin \& Kronberg (1980), centered near $l \approx 255^{\circ}, b \approx 0^{\circ}$, was identified with the Gum nebula by these authors. Structure in RM amplitude has since been noticed in this area in the form of a magnetized shell (Vallée \& Bignell 1983; Stil \& Taylor 
FIG. 1.- Figure available as separate jpg file Correlation between RM and H $\alpha$ intensity from Finkbeiner (2003). Positive RM are indicated by red circles, while negative RMs are indicated by blue circles. Circles are scaled according to RM amplitude.

FIG. 2.- Figure available as separate jpg file Correlation between RM and polarized intensity from Wolleben et al. (2006). See also Wolleben (2007) for a discussion of the large-scale features in this image.

2007) that is also visible in Figure 1. We note also that there is no clear reversal of the sign of RM across the Galactic equator in the area of the Gum nebula.

Region C of Simard-Normandin \& Kronberg (1980) is an area with positive RM bounded by $0^{\circ}<l<60^{\circ}$, $0^{\circ}<b<60^{\circ}$ and was noticed for its wider range in $\mathrm{RM}$ amplitudes from $+50 \mathrm{rad} \mathrm{m}^{-2}$ to $+300 \mathrm{rad} \mathrm{m}^{-2}$. Region $\mathrm{C}$ is in the same area as the bottom of Loop I (a circle with diameter $116^{\circ} \pm 4^{\circ}$ centered around $l=$ $329^{\circ} \pm 1.5, b=+17.5 \pm 3^{\circ}$ Berkhuijsen et al. 1971), but Simard-Normandin \& Kronberg (1980) considered this a coincidence. Figure 1 shows a distinct area inside region $\mathrm{C}$ with consistently high positive $\mathrm{RM}$ in the area $33^{\circ}<$ $l<65^{\circ}, 0^{\circ}<b<40^{\circ}$. The median RM in this area is $66.7 \mathrm{rad} \mathrm{m}^{-2}$, while the median $\mathrm{RM}$ of the adjacent areas $13^{\circ}<l<33^{\circ}$ and $60^{\circ}<l<80^{\circ}$, both with $0^{\circ}<b<40^{\circ}$ is $24.6 \mathrm{rad} \mathrm{m}^{-2}$. The excess $\mathrm{RM}$ amplitude over the immediate surroundings is therefore $42 \mathrm{rad} \mathrm{m}^{-2}$. The high-latitude boundary of this region of enhanced positive RM is not sharp. If it extends well north of $40^{\circ}$ at the level of $\sim 10 \mathrm{rad} \mathrm{m}^{-2}$, it may be responsible for the RM excess associated with the Hercules supercluster by Xu et al. (2006).

Figure 1 also shows enhanced RM amplitude associated with some bright $\mathrm{H}$ II regions, e.g. at $(l, b)=\left(+105^{\circ},+4^{\circ}\right), \quad\left(+78^{\circ},+2^{\circ}\right), \quad\left(+6^{\circ},+23^{\circ}\right) \quad(\zeta$ Oph), $\left(-108^{\circ},-9^{\circ}\right)(\mathrm{Gum})$, and $\left(-165^{\circ},-12^{\circ}\right)(\lambda$ Ori). Smaller excesses of RM amplitude are associated with more diffuse, low-surface brightness $\mathrm{H} \alpha$ emission, e.g. in the Orion-Eridanus region $\left(-180^{\circ}<l<-135^{\circ}\right.$, $-55^{\circ}<b<-5^{\circ}$ ). We also see a small RM excess associated with the shell of the Orion-Eridanus superbubble (Heiles 1976; Brown et al. 1995).

Figure 3 shows RMs on an image of $0.25 \mathrm{keV}$ diffuse X-ray emission from (Snowden et al. 1997). At low latitudes there is little emission because of strong absorption. At high latitudes, emission from the more distant halo is observed, while high-latitude clouds absorb the halo emission locally, and appear as dark silhouettes known as X-ray shadows (Snowden et al. 1997, 1998). Region $\mathrm{C}^{\prime}$ coincides with a large X-ray shadow that has an $\mathrm{HI}$ counterpart at $V_{\mathrm{LSR}}=-2 \mathrm{~km} \mathrm{~s}^{-1}$, and that is part of the outer HI shell of radio loop I (Berkhuijsen et al. 1971).

\section{RM STRUCTURE FUNCTIONS}

Structure in RM on angular scales of tens of degrees becomes visible because of the high sampling density ( $>$ 1 RM per square degree). The RM density on the sky in our data is not sufficient to recognize RM structures on angular scales less than a few degrees, but structure on small angular scales does affect the variance of RM because it introduces differences from one line of sight to another. This addition to the variance of RM may depend on angular scale, and is conveniently expressed as the second-order SF $D$ defined as

$$
D(\delta \theta)=\frac{1}{N} \sum_{i}[R M(\theta)-R M(\theta+\delta \theta)]_{i}^{2},
$$

where the sum is over all pairs of sources with a mutual distance $\delta \theta$ on the sky, and $N$ is the number of pairs included in the sum. The mean distance between an RM measurement and its closest neighbor in our data is 0.65 . SFs can probe RM variance on smaller angular scales than this depending on the number of source pairs available to evaluate the variance with reasonable confidence. For our analysis, the smallest angular scale is taken to be the scale for which we have a minimum of 20 source pairs, or $0 \% 1$, whichever comes first. The largest angular scale we consider for the SFs is $\delta \theta=10^{\circ}$, so the RM gradient related to the large-scale magnetic field does not contribute significantly to D.

All possible sources of variance contribute to the amplitude of the SF. We may write D as a sum of variance terms that we consider statistically independent

$$
D=\sigma_{\text {int }}^{2}+\sigma_{\text {IGM }}^{2}(\delta \theta)+\sigma_{\text {ISM }}^{2}(\delta \theta)+\sigma_{\text {noise }}^{2}(\delta \theta),
$$

with $\sigma_{\text {int }}^{2}$ the variance of RM originating inside the source, $\sigma_{\mathrm{IGM}}^{2}$ the variance in RM originating in the intergalactic medium, for different lines of sight separated by angle $\delta \theta, \sigma_{\text {ISM }}^{2}$ the variance of RM originating in the interstellar medium for lines of sight separated by angle $\delta \theta$, and $\sigma_{\text {noise }}^{2}$ the variance resulting from measurement errors and noise.

The variance $\sigma_{\text {noise }}^{2}$ should be subtracted from $D$ to obtain the sum of the three terms of astrophysical interest (e.g. Haverkorn et al. 2004). This term was calculated from the errors on the RMs contributing to pairs separated by angular distance $\delta \theta$. Each term in the summation of Equation 2 contains differences $R M_{m}-R M_{n}$ ( $m>n$ indices of data in the RM catalog). For each pair of sources included in the summation, the variance added by noise is $\sigma_{m}^{2}+\sigma_{n}^{2}$ where $\sigma_{m}$ and $\sigma_{n}$ are the standard errors for $R M_{m}$ and $R M_{n}$ respectively. This assumes that the error bars in the RM data base provide a realistic estimate of the measurement errors. We defer this discussion to Section 4. In principle $\sigma_{\text {noise }}^{2}$ can depend on $\delta \theta$, but for the SFs presented here, it is virtually constant. The median error in our complete RM catalog is $\sim 10.8 \mathrm{rad} \mathrm{m}^{-2}$, so the error term can be a significant source of variance and it affects the slope of the SF on small angular scales. Unless stated otherwise, SFs in this paper or derived properties such as amplitude and slope have been corrected for variance associated with measurement errors.

Figure [5] shows a simulation of the effect of noise on a SF, and the noise-subtracted SF. The simulated data consisted of 1978 points drawn at random from an image with a random field with input SF with slope 0.6 kindly provided by V. Uritsky. For Figure 5a, measurement errors for each data point were simulated by first drawing a standard deviation from a uniform distribution between 0.4 and 2.0 (data units), then drawing a number from a Gaussian distribution with the chosen standard deviation, to be added to the actual data value. The standard deviation itself was recorded as the error bar for 
FIG. 3.- Figure available as separate jpg file Correlation between RM and $0.25 \mathrm{keV}$ diffuse X-ray intensity from Snowden et al. $(1998)$.

FIG. 4.- Figure available as separate jpg file RM structure on the sky centered at $(l, b)=\left(180^{\circ}, 0^{\circ}\right)$, leaving out data with $|R M|<25 \mathrm{rad} \mathrm{m}^{-2}$, with positive RM in red and negative RM in blue, and no scaling of the circles according to RM amplitude. This representation is analogous to showing a the lowest contour in a radio map at the level of a few sigma. It visualizes RM structure and boundaries where the sign of RM changes. Regions with a high RM amplitude appear more densely sampled. Note the arc-like structures in $\mathrm{RM}$ on angular scales of tens of degrees that add a few hundred $\operatorname{rad}^{2} \mathrm{~m}^{-4}$ to the $\mathrm{SF}$ amplitude on large angular scales.
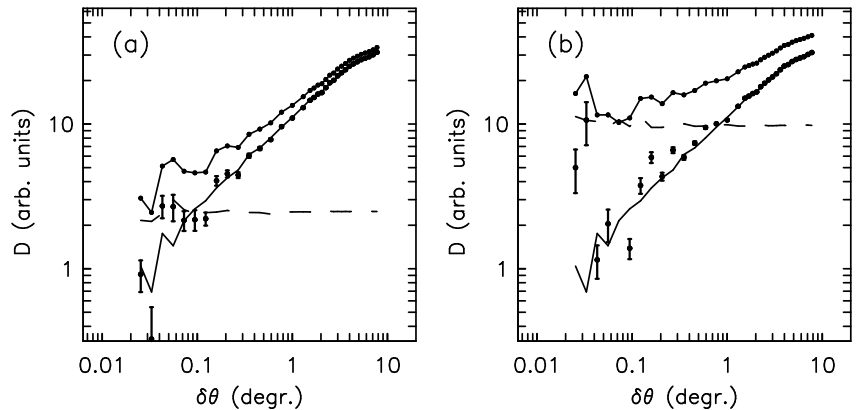

FIG. 5. - Simulation of the effect of noise on a SF, assuming two different levels of RM errors. The upper solid curves with dots represent the measured SFs with noise. The dashed curves show the power calculated from the adopted errors. The points with error bars represent the SF after subtracting the noise power. The SF made with the original 1978 noiseless data points is represented by the lower solid curve. The measurement errors in (b) are on average two times as large as the measurement errors in (a), resulting in a noise power that is 4 times higher in (b). The relative strength of the noise power in (a) is more representative for the data in this paper.

each RM. The figure shows the SF as observed (upper solid curve with dots), the noise power $\sigma_{\text {noise evaluated }}^{2}$ from the error bars of RM (dashed curve), and the SF corrected for noise power (points with error bars), compared with the SF of the same 1978 noiseless data points (lower solid curve). For Figure $5 \mathrm{~b}$, the same set of original data points was used, but the standard deviations of the RM errors were drawn independently from a uniform distribution between 0.8 to 4.0 data units. This on average doubles the measurement errors compared with the situation shown in Figure $5 \mathrm{~b}$, and quadruples the level of the noise power in Equation 3 .

We retrieve the input SF in the simulation shown in Figure [5 and other simulations that included SFs of various slopes and noise levels. These simulations confirmed our evaluation of the noise power even though the errors in individual RMs varied by a factor 5 . On the smallest angular scales, we see occasional large variation in the data after noise subtraction. This is to be expected, because the contribution of the noise to the total variance is large, but our estimate of the noise power is uncertain because relatively few source pairs with small angular separation exist in the catalog. We can easily identify such points and remove them from consideration.

The subtraction of the SF of the noise is a statistical correction that is more accurate for larger samples. The $\mathrm{SF}$ of the measurement errors is not necessarily flat, for instance in the case of periodic variations in the noise of a mosaicking survey with widely separated field centers, or in the case of a deep survey of a small area of the sky where the noise increases towards the edge of the survey area (affecting all source pairs with large angular separation). In our analysis we do not assume that the $\mathrm{SF}$ of the noise is flat, or that all RM error are equal. We calculate the SF of the noise for each $\delta \theta$ from the actual error bars of all included RMs. However, we found no systematic variation of the SF of the noise with angular separation for the Tavlor et al. (2009) RM catalog.

\subsection{Direction dependence of the RM SF}

Before we derive an all-sky image of SF parameters, we examine SFs for a few areas of interest inspired by results discussed in the previous section. These regions visualize the significance of differences in SF parameters with direction. Table 1 lists these regions, and SFs are shown in Figures 6, 7 and 8 ,

Experience from the literature (Haverkorn 2007; Haverkorn et al. 2008; Roy et al. 2008) and from the present data (Stil 2009) shows that the slope of the SF is not always constant, but sometimes changes slope at an angular scale $\delta \theta \approx 1^{\circ}$. The precise angular scale at which a break in the power law occurs is not necessarily the same everywhere, but visual inspection of SFs across the sky suggests that if a break occurs, the SF slope is considerably higher for $\delta \theta<1^{\circ}$ than for $\delta \theta>1^{\circ}$. We therefore fit separate power laws to the SFs at angular scales $\delta \theta \leq 1^{\circ}$ and $\delta \theta>1^{\circ}$ :

$$
D(\delta \theta)=A_{i} \delta \theta^{\alpha_{i}}
$$

with $i=1$ for $\delta \theta \leq 1^{\circ}$ and $i=2$ for $\delta \theta>1^{\circ}$. Table 1 lists results of such fits for the SFs shown in Figures 6, 7 . and 8. The fits for small angular scales are much less constrained because of the limited number of close pairs, and a relatively larger contribution of $\sigma_{\text {noise }}^{2}$ to the total variance on small angular scales. We use the fit parameter $A_{2}$ as a measure of the amplitude of the SF. $A_{2}$ is the variance of $\mathrm{RM}$ for an angular scale of $1^{\circ}$, corrected for variance due to measurement errors.

\subsubsection{Polar regions}

Figure [6]shows RM SFs within 15 degrees of the North Galactic Pole (NGP; 1019 sources) and the South Galactic Pole (SGP; 752 sources). Parameters for these SFs are listed in Table 1. We have fewer RMs in the SGP region because of the declination limit of the NVSS $\left(\delta>-40^{\circ}\right)$, and because of less sky coverage with boths IFs required to derive a RM. The contribution of $\sigma_{\text {noise }}^{2}$ is indicated by the dashed curves, and subtracted in Figure 6 $6 \mathrm{~b}$ and d. Subtracting the noise variance that is approximately independent of angular scale $\delta \theta$, reduces the amplitude of the SF significantly, and also steepens the slope of the SF on small angular scales.

The amplitudes of the SFs at the NGP and the SGP are signifcantly different. The amplitude of the SF in Figure 6 a on angular scales more than $2^{\circ}$ is consistent with the amplitude $\sim 480 \mathrm{rad}^{2} \mathrm{~m}^{-4}$ found by Simonetti, et al. (1984) for this region. These authors did not subtract the $\sigma_{\text {noise }}^{2}$ term in Equation 3, The SF at the NGP also shows a slight increase in amplitude from angular scales 0.2 to 
TABLE 1

Regions and parameters for SFs Shown in Figures 6 and 8

\begin{tabular}{|c|c|c|c|c|c|c|c|c|}
\hline Region & $\stackrel{l_{\min }}{(\text { degr.) }}$ & $\begin{array}{c}l_{\max } \\
(\text { degr. })\end{array}$ & $\begin{array}{c}b_{\min } \\
\text { (degr.) }\end{array}$ & $\begin{array}{c}b_{\max } \\
\text { (degr.) }\end{array}$ & $\log \left(A_{2}\right)$ & $\alpha_{1}$ & $\alpha_{2}$ & Figure \\
\hline NGP & -180 & 180 & 75 & 90 & $2.40(0.03)$ & $0.63(0.39)$ & $0.05(0.04)$ & $6 \mathrm{a}, \mathrm{b}$ \\
\hline SGP & -180 & 180 & -90 & -75 & $2.67(0.03)$ & $0.99(0.58)$ & $0.02(0.04)$ & $6 \mathrm{6}, \mathrm{d}$ \\
\hline Orion & -180 & -135 & -50 & -10 & $2.92(0.02)$ & $0.79(0.08)$ & $0.50(0.03)$ & 7 (black) \\
\hline anti-Orion & -180 & -135 & 10 & 50 & $2.67(0.01)$ & $1.10(0.39)$ & $0.31(0.01)$ & 7 (gray) \\
\hline Region $\mathrm{C}^{\prime}$ & 33 & 68 & 10 & 35 & $3.06(0.01)$ & $0.33(0.20)$ & $0.39(0.02)$ & 7 black) \\
\hline anti-Region $\mathrm{C}^{\prime}$ & 33 & 68 & -35 & -10 & $3.15(0.03)$ & $0.31(0.48)$ & $0.58(0.05)$ & 7 (gray) \\
\hline Region $\mathrm{A}^{\prime}$ & 80 & 150 & -40 & -20 & $2.81(0.01)$ & $0.82(0.30)$ & $0.51(0.02)$ & 7 (black) \\
\hline anti-Region $\mathrm{A}^{\prime}$ & 80 & 150 & 20 & 40 & $2.68(0.01)$ & $0.74(0.26)$ & $0.38(0.03)$ & 7 (gray) \\
\hline Fan & 115 & 160 & 5 & 25 & $2.78(0.02)$ & $-0.02(0.12)$ & $0.58(0.03)$ & 7 (black) \\
\hline anti-Fan & 115 & 160 & -25 & -5 & $2.98(0.02)$ & $0.77(0.17)$ & $0.59(0.04)$ & 7 (gray) \\
\hline Gum & 100 & 100 & 20 & 20 & $3.72(0.04)$ & $1.08(0.41)$ & $1.14(0.09)$ & 8 \\
\hline
\end{tabular}
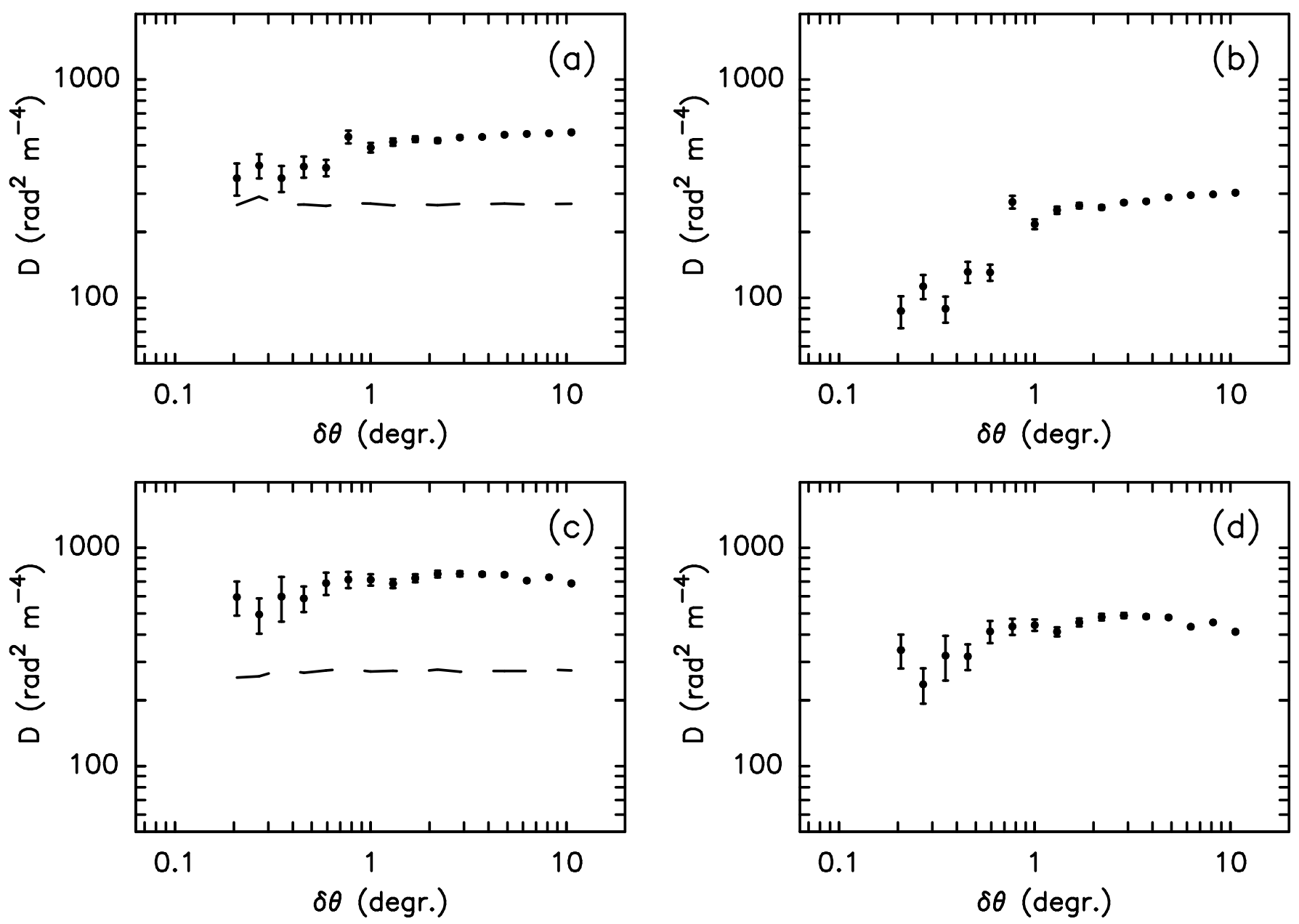

FIG. 6.- (a) RM SF in the NGP region $\left(b>75^{\circ}\right)$. The dashed line gives the power in the SF from measurement errors. (b) SF for the NGP region with noise power subtracted. (c) Same as (a) for the SGP region $\left(b<-75^{\circ}\right)$. (d) Same as (b) for the SGP region.

$10^{\circ}$, but the error of $\alpha_{2}$ in this region is large (Table 11). Mao et al. (2010) reported a slope $0.08 \pm 0.01$ in the NGP region and $0.03 \pm 0.01$ in the SGP region. Our SF at the SGP appears flatter than at the NGP.

A difference in the slope and amplitude of the SFs of the two Galactic pole regions suggests a significant Galactic foreground in these regions. Assuming $\sigma_{\text {IGM }}^{2}$ is negligible, and $\sigma_{\text {int }}^{2}$ is independent of $\delta \theta$ for unrelated sources, a rise in the noise-corrected SF or dif- ferences in amplitude must be related to differences in $\sigma_{\text {ISM }}^{2}$. Since the amplitude of the SF at the SGP is higher than at the NGP, and the SF at the NGP appears to have a positive slope on smaller angular scales, it follows that both Galactic poles have significant foreground RM variations. An upper limit $\sigma_{\text {int }} \lesssim 10 \mathrm{rad} \mathrm{m}^{-2}$ is suggested by the RM variance in the NGP region on angular scales less than $1^{\circ}$. We do not expect $\sigma_{\text {int }}$ to be much less than $5 \mathrm{rad} \mathrm{m}^{-2}$, because the variation of 

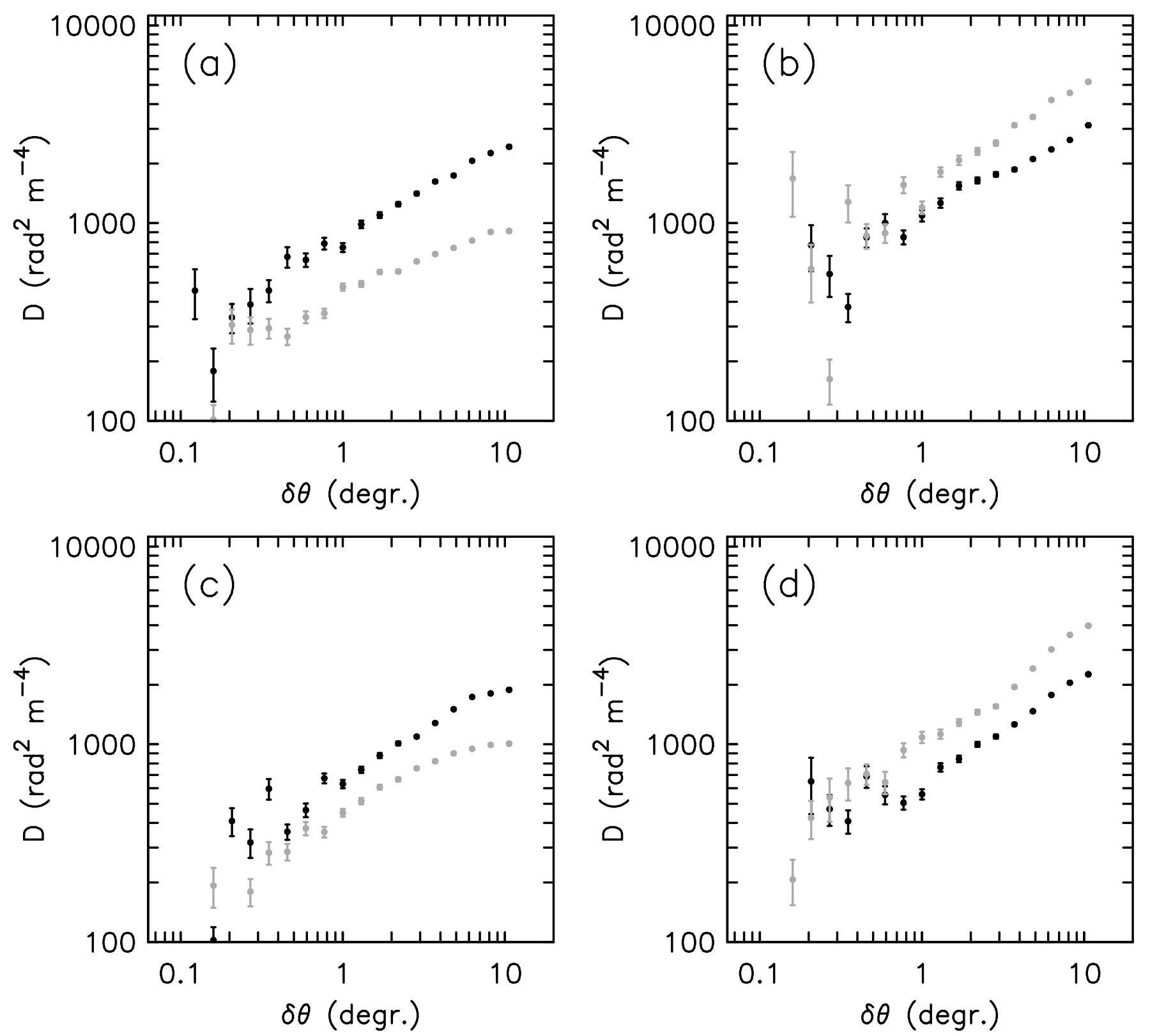

FIG. 7.- (a) RM SF in the Orion region (black) and its northern counter part ("anti-Orion"; grey) as defined in Table 1 The noise variance $\sigma_{\text {noise }}^{2}$ has been subtracted. (b) SF for Region $\mathrm{C}^{\prime}$ (black) and its southern counterpart "anti-region C'" (grey). (c) SF for Region $\mathrm{A}^{\prime}$ (black) and its northern counterpart "anti-region A'" (grey). (d) SF for the Fan region (black) and its southern counterpart "anti-Fan" (grey).

RM across individual sources at high latitude on angular scales less than $1^{\prime}$ (Leahy 1987) is not much smaller than our upper limit determined at angular scales $10^{\prime}-25^{\prime}$ from Figure $6 \mathrm{~b}$, while models of the foreground suggest lower values (Sun \& Reich 2009). We adopt the value $\sigma_{\text {int }}=7 \mathrm{rad} \mathrm{m} \mathrm{m}^{-2}$ and find $\sigma_{\text {ISM }}^{2}=202 \mathrm{rad}^{2} \mathrm{~m}^{-4}$ at the NGP, and $\sigma_{\text {ISM }}^{2}=419 \mathrm{rad}^{2} \mathrm{~m}^{-4}$ at the SGP. If this additional variance arises in a single Faraday screen, the rms RM amplitude of this screen would be $14.7 \mathrm{rad} \mathrm{m}^{-2}$, but there is no evidence that the higher variance results from a separate additional Faraday screen in the southern hemisphere. Taylor et al. (2009) and Mao et al. (2010) reported that the mean RM near near the SGP is larger than near the NGP. The difference in RM variance found here is consistent with the difference in mean RM in the sense that both variance and mean are larger by a factor $\sim 2$ in the south.

\subsubsection{RM SFs at lower latitude}

Figure 7 and Figure 8 show SFs of selected intermediate-latitude areas defined in Table 1. The pairs of SFs in each panel of Figure 7 represent regions with the same longitude, mirrored with respect to the Galactic plane. The Gum region is shown separately in Figure 8 because its mirror region is not covered by the NVSS, and the amplitude of the SF is much larger than the SFs shown in Figure 7. The first impression of Figure 7 is that the amplitude of the SFs is not symmetric with respect to the Galactic plane. The highest SF in each panel of Figure 7 is in the southern Galactic hemisphere, showing 


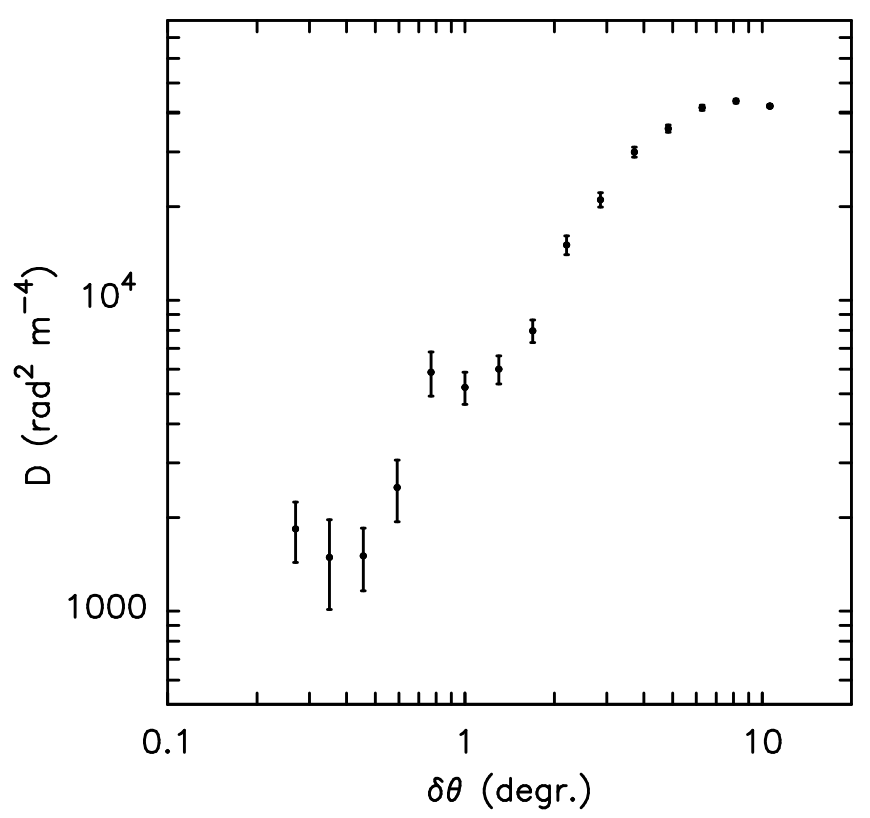

FIG. 8. - SF for the region of the Gum nebula defined in Table 1 .

the same asymmetry as Figure 6. On average these SFs suggest that the variance of $\mathrm{RM}$ in different areas in the southern Galactic hemisphere is $\sim 50 \%$ larger than in the northern Galactic hemisphere. Taylor et al. (2009) found that the mean RM across the southern Galactic hemisphere is consistently higher than in the northern Galactic hemisphere. We will show that the asymmetry in $\mathrm{RM}$ variance is also global.

Figure 7 and Figure 8 also show significant differences in the slope of the SFs. The SF of the Gum region is among the steepest identified in the data. The Orion region has a significantly steeper slope than its counterpart region across the Galactic plane, and the same is true for Region $\mathrm{A}^{\prime}$. In other areas, such as the Fan region, we see a difference in amplitude but no significant difference in slope.

Figure 9 the sky distribution of $A_{2}$ and $\alpha_{2}$ derived from a dense grid of SFs, using sources inside a circular aperture with diameter $15^{\circ}$. The slope and amplitude of the SFs for $\delta \theta>1^{\circ}$ have a similar accuracy as those listed in Table 1, because of the similar number of sources involved. The uncertainty in the amplitude is therefore approximately $5 \%$, and the uncertainty in the slope is approximately 0.03 . Most of the structure visible in Figure 9 represents significant differences in amplitude or slope of the RM SF.

The asymmetry in SF amplitude between the NGP and SGP shown in Figure 6 is also visible in the top panel of Figure 9. There are only a few areas in the south where the amplitude drops below $300 \mathrm{rad}^{2} \mathrm{~m}^{-4}$, while most of the NGP region has amplitudes less than $300 \mathrm{rad}^{2} \mathrm{~m}^{-4}$. Towards the Galactic plane, RM fluctuations increase. The largest fluctuations $\left(4.8 \times 10^{5} \mathrm{rad}^{2} \mathrm{~m}^{-4}\right)$ are found in the Galactic plane, in the direction $l \approx 90^{\circ}$. RM fluctuations may be approximately symmetric with respect to $l=0^{\circ}$, but the declination limit of the NVSS removes the fourth Galactic quadrant and part of the third Galactic quadrant.

The amplitude of the SFs in Figure 9a traces the brightest extended $\mathrm{H} \alpha$ emission, with significantly higher amplitudes towards the inner Galaxy than towards the outer Galaxy. This contrast shows the excentric location of the Sun in the Galaxy, and thus suggests that the low-latitude RM variance on an angular scale of $1^{\circ}$ originates from a line of sight distance of several kpc. Structure at higher latitudes are probably more local in nature. Around longitude $l=-110^{\circ}$, the contours of SF amplitude follow the outline of the Gum nebula in the $\mathrm{H} \alpha$ image. The low-latitude peaks follow bright emission in the Galactic plane, except perhaps the local maximum near $l=40^{\circ}$. The brighter parts of the Orion-Eridanus region $\left(l=-170^{\circ}, b=-40^{\circ}\right)$ are also associated with enhanced RM variance. The outlines of radio Loops IIV (Berkhuijsen et al. 1973) are also shown in Figure 9 . Only Loop III may have have a counterpart in SF amplitude.

The slope of the SF (Figure 9b) is highest in the Galactic plane, but it does not show the same concentration toward the Galactic plane as the amplitude. The largest slopes are $\alpha_{2} \approx 1.3$ in the direction of the $\zeta \mathrm{Oph} \mathrm{H}_{\text {II }}$ region $\left(l=5^{\circ}, b=+24^{\circ}\right)$, and the Gum nebula. At higher northern Galactic latitude, we see a systematic trend that the slope of the SF is shallower towards the Galactic center than towards the Galactic anticenter at the same latitude. The contour of $\alpha_{2}=0.2$ reaches $b \approx 50^{\circ}$ towards $l=0^{\circ}$, while it is found around $b \approx 80^{\circ}$ towards $l=180^{\circ}$. It is not clear whether the same behavior also occurs at southern Galactic latitudes, because of the unsampled region $\delta<-40^{\circ}$. The $\alpha_{2}=0.2$ contour is found mostly between $b=-60^{\circ}$ and $b=-70^{\circ}$, except for $0^{\circ}<l<30^{\circ}$ where it is closer to the Galactic plane similar to its northern counterpart. The over-all distribution of the SF slope at high latitudes is more symmetric with respect to the Galactic plane than the amplitude. There is a suggestion of a steeper slope in the rim of radio Loop III.

Figure 10 shows the amplitude and slope of RM SFs as contours on polarized intensity of diffuse emission at $1.4 \mathrm{GHz}$ from Wolleben et al. (2006). The main features in this polarized intensity were described by Wolleben (2007). The gradual trend in slope if highlatitude SFs with longitude mimics the boundaries of the high-latitude polarized emission described by Wolleben (2007).

In the first Galactic quadrant $\left(0^{\circ}<l<90^{\circ}\right)$, the drop in polarized intensity at $b \approx 30^{\circ}$ and $b \approx-30^{\circ}$ noted by Wolleben et al. (2006) corresponds with RM variance of $600 \pm 100 \mathrm{rad}^{2} \mathrm{~m}^{-4}$ north and south of the Galactic plane, although we see extended polarized emission in some areas with RM variance up to $900 \mathrm{rad}^{2} \mathrm{~m}^{-4}$. The polarized intensity drops abruptly by a factor $\sim 3$ at the boundary, while the average percentage polarization is a factor $\sim 10$ smaller at lower latitudes than for $b>30^{\circ}$ (Wolleben 2007). Some of this difference may result from depolarization at a larger distance. The $23 \mathrm{GHz}$ WMAP polarization image (Page et al. 2007) shows the North Polar Spur polarized at lower latitudes, implying some depolarization occurs for $|b|<30^{\circ}$ at $1.4 \mathrm{GHz}$. We take the abrupt decrease of a factor $\sim 3$ in polarized intensity as a measure for the amount of depolarization by a possible foreground screen.

In the second Galactic quadrant $\left(90^{\circ}<l<180^{\circ}\right)$, 
FIG. 9. - Figure available as separate gif file Direction dependence of SF amplitude $A_{2}$ and slope $\alpha_{2}$. Top: amplitude for $\delta \theta=1^{\circ}$. Contour levels are 300,600,1200, 2400,4800, 9600, and $19200 \mathrm{rad}^{2} \mathrm{~m}^{-4}$. The lowest contours show tickmarks in the direction of smaller amplitude. Grayscales represent $\mathrm{H} \alpha$ intensity in Rayleigh from Finkbeiner (2003) on a logarithmic scale indicated by the color bar. The closed black loop marks the lower southern boundary of the NVSS $\left(\delta=40^{\circ}\right)$, and the gray loops indicate radio loops I-IV from Berkhuijsen et al. (1973)). Bottom: Slope of the SF on scales $>1^{\circ}\left(\alpha_{2}\right)$. Contour levels range from 0.2 to 1.2 with increments 0.2 . Contours at the levels $0.2,0.4$, and 0.8 are labeled. The grayscales are the same as in the upper panel. The SF slope varies between -0.07 and 1.28 .

FIG. 10.- Figure available as separate gif file Same as Figure 9 with gray scales representing polarized intensity of diffuse emission at $1.4 \mathrm{GHz}$ from Wolleben et al. (2006).

the brightest polarized emission is observed at lower latitudes north of the Galactic equator in the Fan region. The brightest polarized emission of the Fan region is intersected by the $900 \mathrm{rad}^{2} \mathrm{~m}^{-4} \mathrm{RM}$ variance contour. In contrast to the first quadrant we do not see a clear anticorrelation between polarized intensity and RM variance, with the possible exception of Radio Loop III.

The third Galactic quadrant $\left(180^{\circ}<l<270^{\circ}\right)$ is only partially sampled by the present data. The region of the Gum nebula as traced by the $600 \mathrm{rad}^{2} \mathrm{~m}^{-4}$ contour appears depolarized. A narrow polarized feature between longitudes $-180^{\circ}>l>-155^{\circ}$, and latitude $-10^{\circ}<b<0^{\circ}$ is an extension of polarized emission in the second quadrant. It does not appear to be correlated with structure in RM variance.

\section{DISCUSSION}

\subsection{RM contributions from the local ISM}

RMs from polarized extragalactic sources probe the entire line of sight from the source to the observer. Most extragalactic sources experience relatively little internal Faraday rotation, and intergalactic Faraday rotation is believed to be substantially smaller than the intrinsic RM variance of the extragalactic sources. With the exception of some sources with high internal Faraday rotation, most of the RM originates from the section of the line of sight inside our own Galaxy. RM amplitude also increases significantly towards the Galactic plane. Although the RM is an integral along the line of sight, a single object can contribute significantly. The Gum nebula (distance $\sim 400 \mathrm{pc}$ Brandt et al. 1971) contributes significantly to the RM, even though the line of sight towards the Gum nebula intersects the inner Galaxy, where the electron density is high over a line of sight of several kpc (Cordes \& Lazio 2002). The Orion star forming region, also at a distance of approximately 400 pc (De Zeeuw et al. 1999), is at higher latitude, so its background is a relatively short line of sight through the Galaxy.

The line of sight through the Galaxy is much shorter for high-latitude sources, and structures in the local ISM may affect the RM significantly. Some high-latitude H II regions were identified as such, consistent with earlier results that showed that individual $\mathrm{H}$ II regions can have a significant impact on RM (Mitra et al. 2003). We have found evidence that Region $\mathrm{C}^{\prime}$ is associated with a nearby cloud revealed by its absorption of soft X-rays from the Galactic halo that is at least partially neutral because it is visible in $\mathrm{H}$ I emission. This associates region $\mathrm{C}^{\prime}$ with the outer HI shell of Loop I. The enhanced RM amplitude in region $\mathrm{C}^{\prime}$ spans only $35^{\circ}$ in Galactic longitude, with its central longitude around $l=45^{\circ}$ (see also Stil \& Tavlor 2007).

Towards the Galactic anticenter, where we expect al- most no contribution from the large-scale Galactic magnetic field, we find curved filamentary structures in RM with amplitudes of a few tens $\mathrm{rad} \mathrm{m}^{-2}$, positive and negative (Figure 4). From Equation 1, we obtain an order-ofmagnitude estimate for the RM imposed by a structure along the line of sight with electron density $n_{e}$, internal regular magnetic field $B$, and line-of-sight extent $l$ :

$$
R M \approx 8\left(\frac{n_{e}}{0.1 \mathrm{~cm}^{-3}}\right)\left(\frac{B}{1 \mu \mathrm{G}}\right)\left(\frac{l}{100 \mathrm{pc}}\right) \mathrm{rad} \mathrm{m}^{-2} .
$$

The RM amplitudes and morphology of these structures resemble one or a superposition of several bubble walls with size of the order of $\sim 100 \mathrm{pc}$ (with a large uncertainty). One of these is the Orion-Eridanus superbubble $\left(170^{\circ}<l<220^{\circ},-30^{\circ}<b<-50^{\circ}\right.$ (Brown et al. 1995), visible in emission in Figure 1 and in absorption in Figure 3. These filaments contribute to the amplitude of RM SFs on large angular scales, increasing the SF slope $\alpha_{2}$.

The overarching picture that emerges is one of RM structure in the local ( $\lesssim$ few kpc) ISM on large scales is mostly associated with bubble walls, H II regions, and local clouds, region $A^{\prime}$ (Table1) being a possible exception. If this is representative for an average few kpc line of sight through the Galaxy, the RM variance added by structures on scales of a few hundred pc should contribute significantly to the total variance in RM at low Galactic latitudes. If bubble walls contribute significantly, models of RM variance should include a correlation between field strength and density on scales of a few hundred pc.

\subsection{Amplitude and slope of SFs}

\subsubsection{Comparison with Mao et al. (2010)}

Mao et al. (2010) derived SFs at the NGP and SGP caps (latitude $|b|>77^{\circ}$ ) based on 354 and $319 \mathrm{RMs}$ respectively. Mao et al. (2010) find almost identical SF amplitudes at both Galactic poles, an apparent contradiction of the result in Figure 6. These authors also presented a comparison of their RMs with our RM catalog, and foud a small correlation coefficient between the two samples (0.39 towards the NGP, and 0.36 towards the SGP). Mao et al. (2010) suggested that the differences between the samples are related to multiple RM components in some sources that cannot be resolved in the two-frequency data of Tavlor et al. (2009), but they did not provide specific examples where this would be the case. While it is true that the two-frequency data in Taylor et al. (2009) cannot resolve non-linearity in the relation between polarization angle and $\lambda^{2}$, rotation measure synthesis with limited $\lambda^{2}$ coverage, as in the sample of Mao et al. (2010) has its limitations (Brentiens \& De Bruvn 2005; Frick et al. 2010). It was recently pointed out by L. Rudnick et al. (unpublished) that RM synthesis can provide erroneous solutions in a 

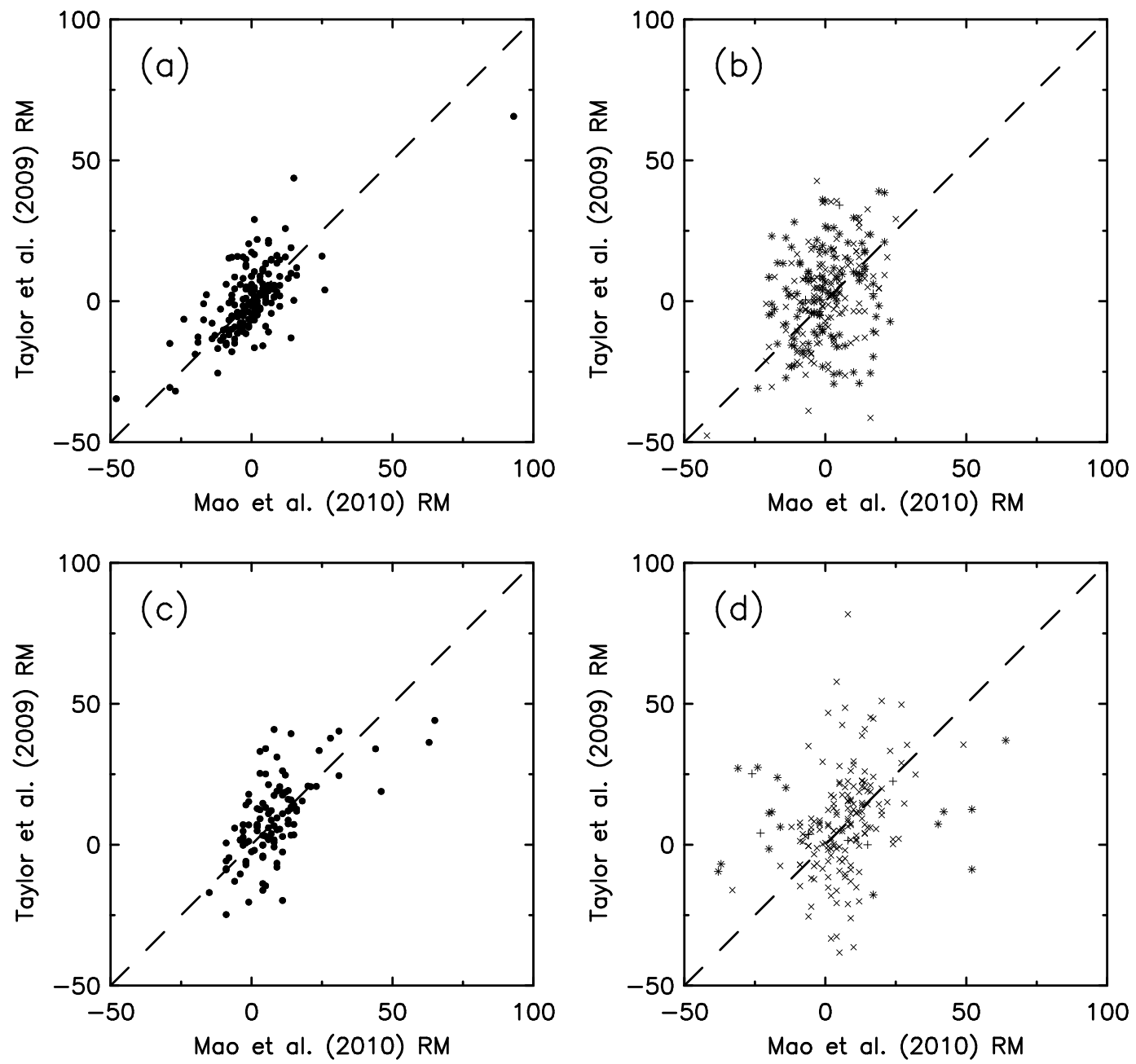

FIG. 11. - Comparison of RMs from Taylor et al. (2009) with RMs from Mao et al. (2010), divided by stated RM error. (a): NGP region with RM error $<8 \mathrm{rad} \mathrm{m}^{-2}$ in both samples. (b): NGP region with RM error $\geq 8 \mathrm{rad} \mathrm{m}^{-2}$ in Tavlor et al. (2009) $(\times)$, and RM error $\geq 8 \mathrm{rad} \mathrm{m}^{-2}$ in Mao et al. (2010) (+). If the errors in both surveys are $\geq 8 \mathrm{rad} \mathrm{m}^{-2}$ both symbols appear, resulting in a $*$. (c): same as (a) for the SGP region. (d): same as (b) for the SGP region.

simple system with just two discrete RM components.

In order to gain a better understanding of the difference between SFs derived by Mao et al. (2010) and those derived here, we must first compare the RM samples in more detail. The median error for sources common to both RM catalogs is $8 \mathrm{rad} \mathrm{m}^{-2}$ in Tavlor et al. (2009), $5 \mathrm{rad} \mathrm{m}^{-2}$ in the NGP region of Mao et al. (2010), and $3 \mathrm{rad} \mathrm{m}^{-2}$ in the SGP region of Mao et al. (2010). Figure 11 shows scatter plots of RMs matched between the two samples, subdivided into RMs with error $<8 \mathrm{rad} \mathrm{m}^{-2}$ (panels a and $\mathrm{c}$ ), and all other RMs (panels $\mathrm{b}$ and $\mathrm{d}$ ).

When considering only RMs with small errors, we see a correlation between the two datasets at both Galactic poles (Figure 11] a and c). The larger scatter in the SGP area found by Mao et al. (2010) is related to RMs with larger error bars, in either Taylor et al. (2009), or Mao et al. (2010), or both. We do not calculate a correlation coefficient, because the correlation coefficient between independent RM surveys of low-RM sources must be small. Unresolved multiple RM components may affect both surveys in a similar way, and thus introduce correlated systematic errors without increasing the scat- ter in Figure 11, but still raise the amplitude of SFs derived from both data sets.

Figure 11 a and c both show significant RM signal. The scatter in these relations provides an estimate of the variance introduced by measurement errors. We examine the subsets with small errors shown in Figure 11 a and c, as well as the complete sample. For the subsets with small errors, the total variance is $90 \mathrm{rad}^{2} \mathrm{~m}^{-4}$ in the north and $135 \mathrm{rad}^{2} \mathrm{~m}^{-4}$ in the south. The error bars (added in quadrature for each source) account for a total variance of $37 \mathrm{rad}^{2} \mathrm{~m}^{-4}$ in Figure 11 and $36 \mathrm{rad}^{2} \mathrm{~m}^{-4}$ in Figure 11]. For the complete sample, the total variance is $151 \mathrm{rad}^{2} \mathrm{~m}^{-4}$ in the north and $193 \mathrm{rad}^{2} \mathrm{~m}^{-4}$ in the south, while the error bars account for $103 \mathrm{rad}^{2} \mathrm{~m}^{-4}$ and $90 \mathrm{rad}^{2} \mathrm{~m}^{-4}$ respectively.

The stated errors underestimate the true variance in the correlation of RMs common to both samples, but more so if the stated errors are small. The RM errors in Taylor et al. (2009) were estimated by error propagation of the noise in the Stokes $Q$ and $U$ images, while the errors in Mao et al. (2010) were estimated as half the width of the RM spread function divided by the signal to noise ratio in polarization. The error estimates in both 
surveys converge to zero as the signal to noise ratio in polarization increases without bound. Perhaps the true errors do not converge as rapidly as assumed. Before we consider the effect of this underestimation of errors on the amplitude of SF at the Galactic poles, we first consider another aspect of the error statistics of the data.

Figure 12 shows the stated errors as a function of RM amplitude for the NGP and SGP regions of the Mao et al. (2010) sample. Black dots represent RMs used in the construction of SFs, and gray crosses respresent rejected RMs. While the median error in Figure 12 a is larger, Figure 12b contains data with large RM errors that contribute to the increased scatter in Figure 11d. RMs with amplitude more than $20 \mathrm{rad} \mathrm{m}^{-2}$ in Figure $12 \mathrm{~b}$ are much more likely to have a large error, introducing a correlation between RM error and RM amplitude: the SGP data of Mao et al. (2010) are heteroskedastic. The highest RM amplitudes (a few percent of the total sample) are rejected in both regions, but the distribution of rejected RMs is more skewed toward high RM amplitude in the south (Figure $12 \mathrm{~b}$ ).

Some RMs in Mao et al. (2010) were rejected in order to eliminate AGNs with strong internal Faraday rotation, and to minimize the effect of known Galactic and extragalactic structures. This approach is preferred if one wishes to determine the intrinsic RM variance of AGNpowered radio sources, or investigate small-scale turbulence at high Galactic latitude. This paper deals with the complete RM foregound. Figure 13 shows the RM errors in relation to RM amplitude for our data in the NGP and $\mathrm{SGP}$ regions. The larger RM errors in our data are compensated by the $\sim 3$ times larger sample size, especially when probing fluctuations on small angular scales.

What does this all mean for the SF amplitudes at the Galactic poles? Figure 14 shows RM SFs at the NGP and SGP. The gray SFs were made with the censored data of Mao et al. (2010) (354 RMs at the NGP and 319 RMs at the SGP). We find a smaller amplitude than Mao et al. (2010), because these authors underestimated the noise power by approximately a factor 2 . The problem was identified and the authors confirmed they recover the SF shown in Figure 14 (A. Mao, private communication). The black points in Figure 14 show SFs made with the complete sample of Mao et al. (2010). The origin of the large scatter for $\delta \theta \lesssim 1^{\circ}$ in Figure 14 a was discussed in Section 3 (Figure [5). Using the complete sample of Mao et al. (2010), we confirm that the SF in the SGP region is a factor $\sim 2$ higher than in the NGP region, but both SFs in Figure 14 have a somewhat smaller amplitude than those in Figure 6] Both data sets suggest a small upward slope of the SF in the NGP region. In Table 1 we list $\alpha_{2}=0.05 \pm 0.04$, while Mao et al. (2010) found $0.08 \pm 0.01$. For the SGP region we find $\alpha_{2}=0.02 \pm 0.04$, while Mao et al. (2010) found $0.03 \pm 0.01$.

If we assume that the noise power in our SFs is really a factor 1.5 higher than that calculated from the RM errors, we would retrieve the black curves in Figure 14. This would be equivalent with the RM errors being underestimated by a factor 1.22 in Tavlor et al. (2009), and account for the variance in the comparison of RMs from the two surveys. While this appears a plausible explanation of the difference, the actual comparison may be more complicated. This analysis assumes that Figure 11 reveals all RM errors, while it does not reveal possible systematic errors common to both surveys that may increase SF amplitude. Another question is the nature of the sources in Mao et al. (2010) for which no reliable RM could be found ( $6 \%$ of their data in the NGP region and $28 \%$ in the SGP region.

It is encouraging that we find consistency between SFs from independent data sets, after accounting for all noise power traced by direct comparison of RMs common to both surveys. This consistency requires just a $22 \%$ increase in the errors quoted by Taylor et al. (2009) (in a low-RM environment), corresponding with only a few rad $\mathrm{m}^{-2}$. It suggests that either the effect of multiple RM components on the data of Taylor et al. (2009) is quite small, or the data from Mao et al. (2010) are affected in the same way for most sources. However, such a systematic error should still increase the amplitude of the SFs, assuming it is uncorrelated between sources. From the total amplitude of the SFs at high latitude, we estimate that any such systematic error should be smaller than $\sim 10 \mathrm{rad} \mathrm{m}^{-2}$.

Our analysis shows that both RM surveys point indicate a larger RM variance in the direction of the SGP than in the direction of the NGP. Our SFs at intermediate Galactic latitude show the same trend, and these are less sensitive to the amplitude of the noise power because it contributes much less to the total variance. Both Taylor et al. (2009) and Mao et al. (2010) reported a higher mean RM in the south than in the north. Tavlor et al. (2009) found this to be the case in the entire southern Galactic hemisphere.

\subsubsection{Distribution of RM variance over the sky}

Variance in RM originates from fluctuations in electron density and magnetic field on a range of scales in the ISM, including $\mathrm{H}$ II regions, bubbles, turbulence, and possibly also molecular clouds with a small ionized fraction but a strong magnetic field. The variance in RM should be approximately proportional to the length of the line of sight through the Galaxy (Sokoloff et al. 1998).

Figure 9 shows that the amplitude of SFs on an angular scale $\delta \theta=1^{\circ}$ follows the distribution of $\mathrm{H} \alpha$ intensity in the Galactic plane. The highest amplitudes at low Galactic latitude are found in the direction of the Local Arm $\left(l \approx 90^{\circ}\right)$ and other regions along the Galactic plane. The peak in $\mathrm{RM}$ variance at $(l, b) \approx\left(40^{\circ}, 0^{\circ}\right)$ occurs in a region of the Galactic plane with strong extinction at visible wavelengths. The enhance variance in the Gum region is clearly extented in Figure 9 with morphology similar to $\mathrm{H} \alpha$ intensity. The low-latitude $\mathrm{RM}$ variance is smaller in the direction of the Galactic anti-center, where the line of sight through the Galaxy is shorter. These observations are consistent with the expectation that variance in RM increases with the length of the line of sight through the Galaxy (Sokoloff et al. 1998). Higher electron density and stronger turbulence related to more intense star formation in the inner Galaxy probably also contribute to the high RM variance for lines of sight through the inner Galaxy. The outer scale in RM variance associated with interarm regions, but not with spiral arms reported by Haverkorn (2007) and Haverkorn et al. (2008) indicates that besides line-of-sight distance, the local conditions are also important.

The difference in RM variance between the Galactic 

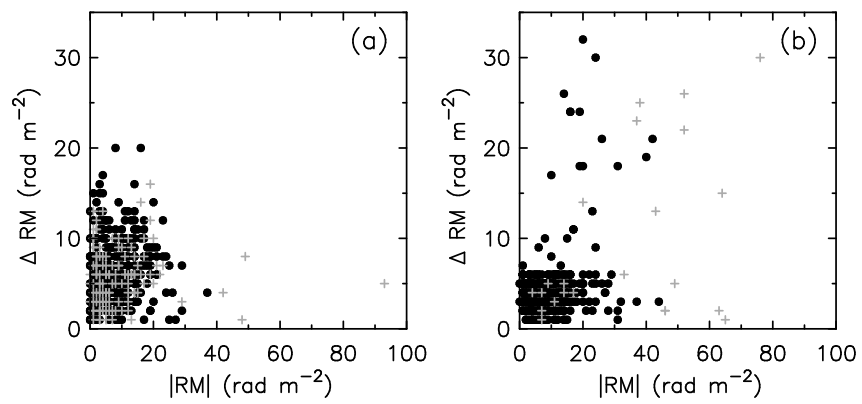

FIG. 12. - Correlation of RM error with RM amplitude for the sample of Mao et al. (2010). (a): NGP region observed with the WSRT, (b): SGP observed with the ATCA. Black dots represent RMs used in the SF by Mao et al. (2010) (heavily saturated by overlapping symbols in some parts of the figure). The gray crosses represent data rejected by Mao et al. (2010) for use in SFs.

northern and southern hemispheres (Figure 9 and Table 11) is particularly interesting. Assuming the intrinsic $\mathrm{RM}$ variance of extragalactic sources to be $\sigma_{\text {int }}=$ $7 \mathrm{rad} \mathrm{m}^{-2}$ (Equation 3), the RM variance introduced by the Galactic foreground is $202 \mathrm{rad}^{2} \mathrm{~m}^{-4}$ at the NGP, and $419 \mathrm{rad}^{2} \mathrm{~m}^{-4}$ at the SGP $\left(120 \mathrm{rad}^{2} \mathrm{~m}^{-4}\right.$ and 307 $\mathrm{rad}^{2} \mathrm{~m}^{-4}$ respectively using SFs from Figure 14). The $\mathrm{RM}$ variance near the SGP originating from the Galactic foreground is therefore a factor 2 larger than the Galactic RM variance near the NGP. The difference in variance is equivalent with an additional source of $R M$ variation with amplitude $14 \mathrm{rad} \mathrm{m}^{-2}$ in the southern Galactic hemisphere. The Sun is located 20 pc from the Galactic mid-plane in the direction of the NGP. This distance is too small compared with the scale height of the ionized gas to make a significant difference in $\mathrm{RM}$ variance. It is more likely that we see a local asymmetry of density or magnetic field, or both with respect to the Galactic plane. Taylor et al. (2009) also found a factor $\sim 2$ difference in the mean RM of the NGP and the SGP, while Mao et al. (2010) found a significant positive RM at the SGP but not at the NGP.

The slope of the SF is higher near the Galactic plane with most values in the range from 0.4 to 0.8 and excesses up to 1.3 in the direction of two nearby $\mathrm{H}$ II regions ( $\zeta$ Oph and the Gum nebula). The slope of our SFs (Table 1) is generally smaller than the $5 / 3$ slope expected for Kolmogorov turbulence (Armstrong et al. 1995; Minter \& Spangler 1996), to the smallest angular scales probed by our data $(\sim 0.3)$. Beck (2007) found $\mathrm{RM}$ SF slopes $\sim 0.3$ in the nearly face-on galaxy NGC 6946 on scales up to $6 \mathrm{kpc}$, which is in the range of values of $\alpha_{2}$ in Table 1

If a change in slope occurs in the range of angular scales probed by the current data $\left(0.1<\delta \theta<10^{\circ}\right)$, the slope on larger angular scales is usually smaller. A change in SF slope is sometimes interpreted as a physical scale in the turbulence probed by RM variance, that could be an outer scale length if the SF becomes flat on large scales. An implicit assumption is that the RM variance traces stochastic variations of electron density and magnetic field related to turbulence in the magneto-ionized ISM. If deterministic structure, e.g. an $\mathrm{H}$ II region that may or may not be detectable in current $\mathrm{H} \alpha$ surveys, or a meso-scale structure in the magnetic field, contributes significantly to the variation of RM with position over the area for which the SF is evaluated, the shape of the SF
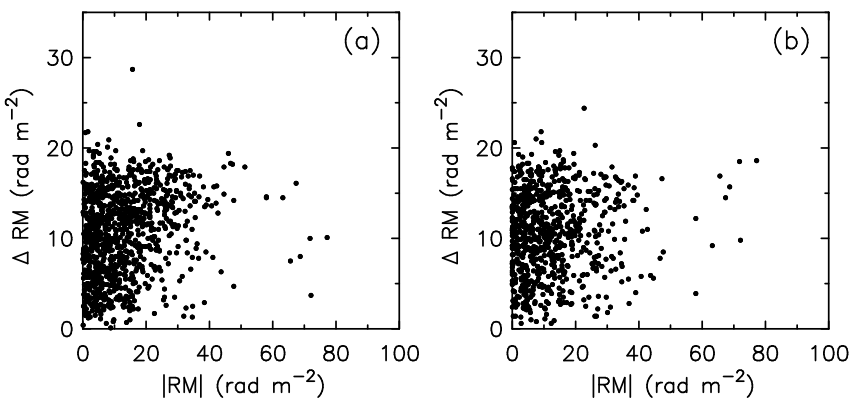

FIG. 13. - Same as Figure 12 for the data from Taylor et al. (2009). (a) NGP region. (b) SGP region.

may reflect the angular scale of the ionized structure, not the turbulence along the line of sight. The present data suggest that this may be true in some directions. The steepest SFs at low latitude are associated with bright extended $\mathrm{H}$ II regions in Figure 9, Figure 4 also shows $\mathrm{RM}$ structure on angular scales $\gtrsim 10^{\circ}$ with amplitude of a few tens of $\mathrm{rad} \mathrm{m} \mathrm{m}^{-2}$, sufficient to affect the slope of the $\mathrm{SF}$ between $\delta \theta=1^{\circ}$ and $\delta \theta=10^{\circ}$. Sampling with a denser RM grid can reveal such structures, as in the case of the RM anomaly in Cygnus (Whiting et al.|2009). Future wide-area RM surveys with the Arecibo radio telescope (GALGACTS), the Australian SKA Pathfinder ASKAP (POSSUM), and with the Square Kilometre Array will provide denser sampling that may reveal underlying structure that affects the slope of our SFs, while not recognizable in the present data.

Model SFs by Sun et al. (2008) and Sun \& Reich (2009) that assume a turbulent Kolmogorov power spectrum for density and magnetic field, predict a flat SF at low latitude on angular scales $\delta \theta \gtrsim 3^{\prime}$ as the number of turbulent cells in the line of sight increases. These models explore angular scales much smaller than those probed here. We see a general increase in SF slope towards the Galactic plane, contrary to the expectation of a smaller slope expected by Sun \& Reich (2009). As noted in Figure 9, the lack of a strong longitude dependence of SF slope, and its wider distribution with Galactic latitude suggest that the slope of SFs on larger angular scales is affected by the local interstellar medium. This suggests that local structure not represented in the models of Sun \& Reich (2009) may be responsible for the steep slope of the SFs at low latitude.

\subsection{Depolarization of diffuse emission}

RMs s of extragalactic sources are affected by Faraday rotation along the entire line of sight from the source to the observer, but mostly in the Galaxy. Depolarization of diffuse emission extends to $b \approx 30^{\circ}$. At this latitude, the line of sight through the Galactic ISM is approximately twice as long as the line of sight towards the Galactic poles. Adopting a scale height of the warm ionized medium $H=1.83_{-0.25}^{+0.12} \mathrm{kpc}$ (Gaensler et al. 2008), most of the Faraday rotation in a line of sight at $b=30^{\circ}$ should arise within a distance $\sim 3.6 \mathrm{kpc}$. This is still more than an order of magnitude larger than the distance to the North Polar Spur, for which we adopt the distance to the Scorpio-Centaurus OB association (170pc; Egger \& Aschenbach 1995), although the near side may be closer. Assuming an exponential density profile, and $\delta n_{e} \sim n_{e}, \delta B \sim B$ independent of density, approximately 

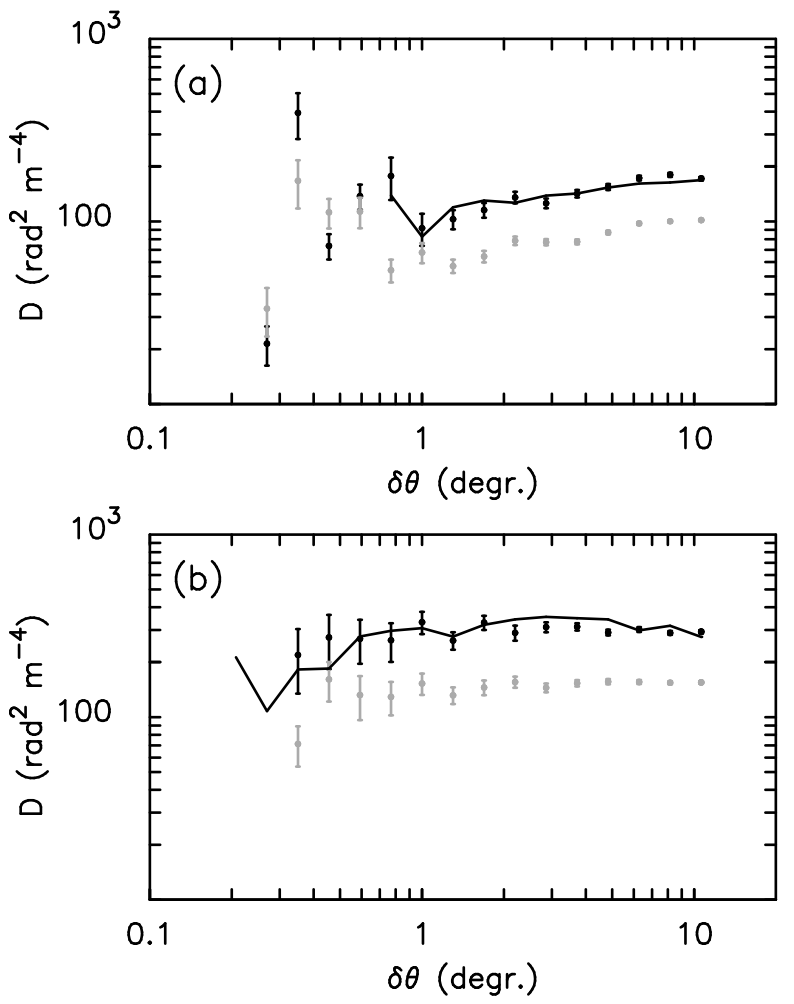

FIG. 14.- SFs at the NGP (a) and SGP (b) regions, comparing results obtained with data from Taylor et al. (2009) and Mao et al. (2010). Gray points are SFs made with RMs from Mao et al. (2010), using 354 accepted RMs in the north and 319 accepted RMs in the south. These SFs differ from Figure 6 in Mao et al. (2010) as discussed in the text. The black dots represent SFs that include all RMs in Mao et al. (2010). The black curves represent the SFs from this paper (Figure 6) with the noise power multiplied by 1.5 before subtraction. The black curve in panel (a) does not extend to angular scales smaller than 0.7 because the noise power times 1.5 is comparable to the total variance of the data.

half of the Faraday rotation and RM variance occurs in front of the North Polar Spur. Large-scale inhomogeneity of the ISM introduces significant uncertainty in this estimate.

A foreground screen with $\mathrm{RM}$ variance $\sigma_{\mathrm{RM}}^{2}$ on scales much smaller than the 0 . beam size of the DRAO $26-\mathrm{m}$ telescope depolarizes emission observed at wavelength $\lambda$ by a factor

$$
\frac{p}{p_{0}}=\exp \left(-2 \sigma_{\mathrm{RM}}^{2} \lambda^{4}\right),
$$

(Sokoloff et al. 1998). The sudden drop of a factor $\sim 3$ in polarized intensity at $|b|=30^{\circ}$ observed at $\lambda=21 \mathrm{~cm}$ requires $\sigma_{\mathrm{RM}}^{2}=280 \pm 180 \mathrm{rad}^{2} \mathrm{~m}^{-4}$, where the error represents a factor 2 uncertainty in the depolarization factor. The observed total RM variance is $600 \pm 100 \mathrm{rad}^{2} \mathrm{~m}^{-4}$. Around the depolarization edge, the SF slope is between 0.3 and 0.4 (Figure 10). Assuming a typical slope 0.35 around $|b|=30^{\circ}$, we find the observed $\mathrm{RM}$ variance on the scale of the beam of the DRAO 26-m telescope $(470 \pm 100) \mathrm{rad}^{2} \mathrm{~m}^{-4}$. Subtracting the variance related to measurement errors $\sigma_{\text {noise }}^{2}=270 \pm 18 \mathrm{rad}^{2} \mathrm{~m}^{-4}$ and the intrinsic variance of background sources $\sigma_{\text {int }}^{2}=$
$49 \pm 20 \mathrm{rad}^{2} \mathrm{~m}^{-4}$ (Equation 3), the RM variance attributed to the Galactic ISM along the line of sight is $150 \pm 110 \mathrm{rad}^{2} \mathrm{~m}^{-4}$. Approximately half of this variance is expected to arise behind the North Polar Spur, and would not depolarize emission from this region.

The observed variance is a factor $\sim 3$ lower than what is required to depolarize the diffuse emission, but in view of the estimated errors, the difference is not very significant. Within the errors, it is possibile that differential Faraday rotation along the line of sight contributes to depolarization of the diffuse emission.

\section{SUMMARY AND CONCLUSIONS}

This paper presents an analysis of structure in the magneto-ionized interstellar medium probed by 37,543 RMs derived from the NVSS (Tavlor et al. 2009). RM SFs are presented as a function of direction for $80 \%$ of the sky. We find that:

1. Large-scale structures visible in RM amplitude and sign appear related to structures in the Local ISM, such as radio Loops I and II, the Gum nebula and some other $\mathrm{H}$ II regions. We associate region $\mathrm{C}^{\prime}$ (Table 1) with a soft $\mathrm{X}$-ray shadow also visible in $\mathrm{H}$ I at $V_{\mathrm{LSR}} \approx-2 \mathrm{~km} \mathrm{~s}^{-1}$.

2. SFs near the Galactic poles indicate the presence of a Faraday screen at both Galactic poles. The variance in RM from foregound ISM is $\sigma_{\text {ISM }}^{2}=202 \mathrm{rad}^{2} \mathrm{~m}^{-4}$ and the NGP, and $\sigma_{\text {ISM }}^{2}=419 \mathrm{rad}^{2} \mathrm{~m}^{-4}$ at the SGP $\left(120 \mathrm{rad}^{2} \mathrm{~m}^{-4}\right.$ and $307 \mathrm{rad}^{2} \mathrm{~m}^{-4}$ respectively when using Figure 14). This difference is attributed to a local asymmetry of electron density and/or properties of the magnetic field with respect to the Galactic plane.

3. The distribution of RM variance over the sky shows a systematic trend with Galactic longitude such that lines of sight through the inner Galaxy have higher RM variance than lines of sight through the outer Galaxy. This suggests that most or all of the line of sight through the Galaxy contributes to RM variance. The slope of the $\mathrm{SF}$ does not show the same trends, suggesting its origin is related to local structures that dominate RM variance on larger angular scales.

4. Radio Loop III and the Orion-Eridanus bubble appear to enhance $\mathrm{RM}$ variance between latitudes $30^{\circ}$ and $50^{\circ}$, and a higher SF slope is found in these areas. Several other curved filamentary structures are found in direction of the Galactic anti-center (Figure 4).

5 . The steepest SFs $\left(\alpha_{2} \approx 1.3\right)$ are found in the direction of the Gum nebula and the $\zeta$ Oph H II regions, but the steep slope may be a result of a localized enhancement of the mean RM by these two $\mathrm{H}$ II regions.

6. RM variance traced by compact extragalactic radio sources can account for depolarization of diffuse Galactic emission given the uncertainties, but the over-all variance is on the low side allowed by the errors. Differential Faraday rotation along the line of sight may increase depolarization as well.

JMS thanks dr. Vadim Uritsky for providing a set of images with different SF parameters as part of a blind test of the SF analysis. This research was supported by a Discovery Grant of the Natural Sciences and Engineering Research Council of Canada to Jeroen Stil. 


\section{REFERENCES}

Armstrong, J. W., Rickett, B. J., \& Spangler, S. R. 1995, ApJ, 443,209

Axon, D. J., \& Ellis, R. S. 1976, MNRAS, 177, 499

Beck, R. Shukurov, A., Sokoloff, D., \& Wielebinsky, R. 2003, A\&A, 411, 99

Beck, R. 2007, A\&A, 470, 539

Berdyugin, A., \& Teerikorpi, P. 2002, A\&A, 384, 1050

Berdyugin, A., Piirola, V., \& Teerikorpi, P. 2004, A\&A, 424, 873

Berkhuijsen, E. M., Haslam, C. G. T. \& Salter, C. J. 1971, A\&A, 14,252

Berkhuijsen, E. M. 1973, A\&A, 24, 143

Brandt, J. C., Stecher, T. P., Crawford, D. L., \& Maran, S. P. 1971, ApJ, 163, 99

Brentjens, M. A., \& De Bruyn, A. G. 2005, A\&A 441, 1217

Brown, A. G. A., Hartmann, D., \& Burton, W. B. 1995, A\&A, 300, 903

Brown, J. C., \& Taylor, A. R. 2001, ApJ, 563, L31

Brown, J. C., Taylor, A. R., Wielebinski, R., \& Müller, P. 2003, ApJ, 592, L29

Brown, J. C., Haverkorn, M., Gaensler, B. M., Taylor, A. R. Bizunok, N. S., McClure-Griffiths, N. M., Dickey, J. M., \& Green, A. J. 2007, ApJ 663, 258

Burn, B. J. 1966, MNRAS, 133, 67

Condon, J. J., Cotton, W. D., Greisen, E. W., Yin, Q. F., Perley, R. A., Taylor, G. B., \& Broderick, J. J. 1998, AJ, 115, 1693

Cordes, J. M., \& Lazio, T. J. 2002, astro-ph/0207156

De Bruyn, A. G., Bernardi, G. \& the LOFAR collaboration 2009, in The Low-Frequency Radio Universe ASP Conference Series, Vol. 407, proceedings of the conference held 8-12 December 2008, at National Centre for Radio Astrophysics (NCRA), TIFR, Pune, India. Edited by D. J. Saikia, D. A. Green, Y. Gupta, and T. Venturi. San Francisco: Astronomical Society of the Pacific, 3

De Zeeuw, P. T., Hoogerwerf, R., De Bruijne, J. H. J., Brown, A. G. A., \& Blaauw, A. 1999, AJ, 117, 354

Dineen, P., \& Coles, P., MNRAS, 362, 403

Duncan, A. R., Haynes, R. F., Jones, K. L., \& Stewart, R. T. 1997, MNRAS, 291, 279

Duncan, A. R., Reich, P., Reich, W., \& Fürst, E. 1999, A\&A, 350,447

Egger, R. J., \& Aschenbach B. 1995, A\&A, 294, L25

Finkbeiner, D. 2003, ApJS, 146, 407

Frick, P., Stepanov, R., Shukurov, A., Sokoloff, D. 2001, MNRAS, 325, 649

Frick, P., Sokoloff, D., Stepanov, R., \& Beck, R. 2010, MNRAS, 401, L24

Gaensler, B. M., Dickey, J. M., McClure-Griffiths, N. M., Gr een, A. J., Wieringa, M. H., \& Haynes, R. F. 2001, ApJ, 549, 959

Gaensler, B., Haverkorn, M., Staveley-Smith, L., Dickey, J. M., McClure-Griffith, N. M., Dickel, J. R., \& Wolleben, M. 2005, Science, 307, 1610

Gaensler, B., Madsen, S., Chatterjee, S., \& Mao, S. A. 2008, PASA, 25, 184

Gao, X. Y., Reich, W., Han, J. L., Sun, X. H., Wielebinski, R., Shi, W. B., Xiao, L., Reich, P., Fürst, E., Chen, M. Z., \& Ma, J. 2010, A\&A, 515, 64

Gray, A. D., Landecker, T. L., Dewdney, P. E. \& Taylor, A. R. 1998, Nat., 393, 660

Han, J. L., Manchester, R. N., Lyne, A. G., Qiao, G. J., \& Van Straten, W. 2006, ApJ, 642, 868

Haverkorn, M., Katgert, P., \& De Bruyn, A. G. 2003, A\&A, 403, 1045

Haverkorn, M., Gaensler, B. M., McClure-Griffiths, N. M., Dickey, J. M., \& Green, A. J. 2004, ApJ, 609, 776

Haverkorn, M., Gaensler, B., Brown, J. C., Bizunok, N. S., McClure-Griffiths, N. M., Dickey, J. M., \& Green, A. J. 2006, ApJ, 637, L33

Haverkorn, M. 2007, in SINS - Small Ionized and Neutral Structures in the Diffuse Interstellar Medium ASP Conference Series, Vol. 365, Proceedings of the conference held 21-24 May, 2006 at the National Radio Astronomy Observatory in Socorro, New Mexico, USA. Edited by M. Haverkorn and W. M. Goss. San Francisco: Astronomical Society of the Pacific, 242
Haverkorn, M., Brown, J. C., Gaensler, B. M., \&

McClure-Griffiths, N. M. 2008, ApJ, 680, 362

Heiles, C. 1976, ApJ, 208, L137

Heiles, C. 2000, AJ, 119, 923

Johnston-Hollitt, M., Hollitt, C. P., \& Ekers, R. D. 2004, in The Magnetized Interstellar Medium, Proceedings of the conference, held in Antalya, Turkey, September 8 - 12, 2003, Eds: B. Uyaniker, W. Reich, and R. Wielebinski, Copernicus GmbH, Katlenburg-Lindau., 13

Leahy, J. P. 1987, MNRAS, 226, 433

Leroy, J. L., 1999, A\&A, 346, 955

Mao, S. A., Gaensler, B. M., Haverkorn, M., Zweibel, E. G., Madsen, G. J., McClure-Griffiths, N. M., Shukurov, A., \& Kronberg, P. P. 2010, ApJ, 714, 1170

Mathewson, D. S., \& Ford, V. L. 1970, MmRAS, 74, 139

Men, H., Ferrière, K., Han, J. L. 2008, A\&A, 486, 819

Minter, A. H., \& Spangler, S. R. 1996, ApJ, 458, 194

Mitra, D., Wielebinski, R., Kramer, M., \& Jessner, A. 2003, A\&A, 398, 993

Page, L., Hinshaw, G., Komatsu, E., Nolta, M. R., Spergel, D. N. et al. 2007, ApJS, 170, 335

Roy, S., Rao, A. P., \& Subrahmanyan, R. 2008, A\&A, 478, 435

Schnitzeler, D. H. F. M., Katgert, P., Haverkorn, M. \& De Bruyn, A. G. 2007, A\&A, 461, 963

Snowden, S. L., Egger, R., Freyberg, M. J., McCammon, D., Plucinsky, P. P., Sanders, W. T., Schmitt, J. H. M. M. Trümper, J., \& Voges, W. 1997, ApJ, 485, 125

Snowden, S. L., Egger, R., Finkbeiner, D. P., Freyberg, M. J., \& Plucinsky, P. P. 1998, ApJ, 493, 715

Simonetti, J. H., Cordes, J. M., \& Spangler, S. R. 1984, ApJ, 284, 126

Simonetti, J. H., Cordes, J. M. 1986, ApJ, 310, 160

Simonetti, J. H. 1992, ApJ, 386, 170

Simard-Normandin, M., \& Kronberg, P. P. 1979, Nat. 279, 115

Simard-Normandin, M., \& Kronberg, P. P. 1980, ApJ, 242, 74

Sokoloff, D. D., Bykov, A. A., Shukurov, A., Berkhuijsen, E. M., Beck, R., \& Poezd, A. D. 1998, MNRAS, 299, 189

Stil, J. M., \& Taylor, A. R. 2007, ApJ, 663, L21

Stil, J. M. 2009, PoS(PRA2009)045

Sun, X. H., \& Han, J. L. 2004, in The Magnetized Interstellar Medium, Proceedings of the conference, held in Antalya, Turkey, September 8 - 12, 2003, Eds: B. Uyaniker, W. Reich, and R. Wielebinski, Copernicus GmbH, Katlenburg-Lindau., 25

Sun, X. H., Reich, W., Waelkens, A. \& Ensslin, T. A. 2008, A\&A, 477,573

Sun, X. H., \& Reich, W. 2009, A\&A, 507, 1087

Taylor, A. R., Gibson, S. J., Peracaula, M., Martin, P. G., Landecker, T. L., Brunt, C. M., Dewdney, P. E., Dougherty, S. M., Gray, A. D., Higgs, L. A., Kerton, C. R., Knee, L. B. G., Kothes, R., Purton, C. R., Uyaniker, B., Wallace, B. J., Willis, A. G., \& Durand, D. 2003, AJ, 125, 3145

Taylor, A. R., Stil, J. M. \& Sunstrum, C. 2009, ApJ, 702, 1230

Uyaniker, B., Fürst, E., Reich, W., Reich, P., \& Wielebinski, R. 1999, A\&AS, 138, 31

Uyaniker, B., Landecker, T. L., Gray, A. D., \& Kothes, R. 2003, ApJ, 585, 785

Vallée, J. P. 2008, ApJ, 681, 303

Vallée, J. P., \& Bignell, R. C. 1983, ApJ, 272, 131

Whiting, C. A., Spangler, S. R., Ingleby, L. D., \& Haffner, L. M. 2009, ApJ, 694, 1452

Wolleben, M., Landecker, T. L., Reich, W., \& Wielebinski, R. 2006, A\&A, 448, 411

Wolleben, M. 2007, ApJ, 664, 349

Xu, Y., Kronberg, P. P., Habib, S., \& Dufton, Q. W. 2006, ApJ, 637,19 
This figure "f1.jpg" is available in "jpg" format from: http://arxiv.org/ps/1010.5299v1 
This figure "f2.jpg" is available in "jpg" format from: http://arxiv.org/ps/1010.5299v1 
This figure "f3.jpg" is available in "jpg" format from: http://arxiv.org/ps/1010.5299v1 
This figure "f4.jpg" is available in "jpg" format from: http://arxiv.org/ps/1010.5299v1 
This figure "f9.gif" is available in "gif" format from: http://arxiv.org/ps/1010.5299v1 
This figure "f10.gif" is available in "gif" format from: http://arxiv.org/ps/1010.5299v1 